\section{SIMINDEX: A FORTRAN 77 program for converting sort data into similarity matrices}

\author{
Dale K. Tiller and Peter K. Lunt \\ University of Oxford, Oxford, United Kingdom
}

There has been an increase in the popularity of cluster analysis and multidimensional scaling techniques for assessing the degree of perceived relationship among objects and/or concepts. Although a number of methods are available for assessing inter-object or concept similarity, the stimulus sorting method, in which subjects sort a set of stimuli into as many groups as they wish, appeals to many researchers. This sorting task is easy for subjects to understand and complete, and involves much less work than directly rating the similarity of all possible pairs of objects.

Having collected a set of similarities data using the stimulus sorting method, the researcher faces the onerous and potentially errorprone task of converting these data into a stimuli $\times$ stimuli similarity matrix. Several programs for producing similarity matrices operate in batch mode on a file containing the sort data, which the user must prepare before running the program (Greenberg \& McIsaac, 1984; Takane, 1981). In contrast, SIMINDEX is an interactive program that allows the user to directly input the names of objects grouped together by individual subjects. From this input, the program creates a stimuli $\times$ stimuli co-occurrence matrix, in which the value of each cell $(i, j)$ in the matrix is the number of subjects sorting objects $i$ and $j$ into the same group.

Description. The similarities matrix is created in the following manner. For each subject, the number of iterations the program performs equals the number of groups formed by that subject. For each group, the user enters the names of the constituent objects at the terminal. Each

This work was supported by a Natural Sciences and Engineering Research Council of Canada Postgraduate Scholarship and a British ORS award to the first author, and an ESRC award to the second author. Correspondence should be addressed to either author at the Department of Experimental Psychology, South Parks Rd., Oxford OX1 3UD, United Kingdom. object name is compared with the contents of a dictionary file containing the names of all the objects. The ordinal position in this dictionary of each object's name in any respective group is then stored in a one-dimensional array. All cells $(i, j)$ in the similarities matrix defined by all the possible paired combinations of values stored in this one-dimensional array are then incremented by one. This process is repeated for each group formed by each subject.

Input. Primary input is from the terminal. The user responds to specific queries from the program (e.g., number of subjects, number of groups per subject, number of objects per group), and also enters the names of the objects in each group. Secondary input to the program is from two other sources. A stimuli $\times$ stimuli data matrix is read and stored each time the program is run. In addition, a file which contains the names of all the objects and functions as the dictionary file is required.

Output. After each subject's sort data have been entered, and at the completion of the session, the program outputs either a square or lower triangular stimuli $x$ stimuli similarities matrix to a disk file. When each successive subject's data have been entered, any previous versions of the output data file currently on disk are overwritten; however, the program is easily modified so that a separate similarity matrix is written to disk for each subject.

Language and Computer. SIMINDEX is written in FORTRAN 77, and was developed and implemented on a Digital VAX 11/780 machine. One nonstandard FORTRAN statement is used in SIMINDEX, involving a variable in a format list, which is easily modified. Thus, the program is compatible with virtually all FORTRAN compilers.

Availability. A listing of the program, and a copy of the program users' manual, are available without charge from either author.

\section{REFERENCES}

GreenberG, E. A., McIsAaC, M. S. (1984). SIMATRIX: A program using the SAS system for multidimensional scaling analysis of sorting data. Behavior Research Methods, Instruments \& Computers, 16, 407-408.

TAKANE, Y. (1981). MDSORT: A special purpose multidimensional scaling program for sorting data. Behavior Research Methods \& Instrumentation, 13, 698. 